\title{
Data Acquisition and Pretreatment of Gastric Magnetic Signal Based on the Giant Magneto Impedance Effect
}

\author{
A.N. Wang, Z.B. Yu, C. Zhang, Z.J. Huang \\ College of Information Science and Engineering \\ Northeastern University \\ Shen Yang, China
}

\begin{abstract}
Surface gastric electromagnetic signal is an important basis for the study of gastrointestinal dynamics. Surface electrode method can only collects the gastric slow wave which is the characterization of gastric contraction rhythm. But it can collect the gastric electrical fast signal which is the characterization of the contraction strength of stomach. Because the fat layer has the filtering effect on gastric electric signal. And the permeability of the fat layer is approximate to 1 , the feature of gastric electrical fast wave can be acquired by measuring the gastric magnetic signal. To solve this problem, this paper puts forward a method of using the sensor base on the giant magneto impedance effect (GMI) to measure the gastric magnetic signal. We use GMI sensor, and design a gastric magnetic signal collection system, according to the actual data analysis of the noise sources, and put forward gastric magnetic signal de-noising algorithms based on wavelet analysis and chaos theory to remove the corresponding noise, which provides the basis for the extraction of gastric electrical fast wave signal.
\end{abstract}

Keywords-GMI; gastric electromagnetic signal; labview; chaos algorithm

\section{INTRODUCTION}

Up to $20 \% \sim 40 \%$ of people around the world suffer from stomach functional disease [1]; functional gastric diseases affect people's physical and mental health seriously. Gastric electrical signals can be used as an important basis for the diagnosis of gastric function; it is divided into gastric electrical slow wave and gastric electrical fast wave. Gastric electrical slow wave is reflected in the contraction rhythm of stomach, gastric electric fast wave is reflected in the strength of contraction of the stomach. Get gastric electrical slow wave and gastric electrical fast wave information at the same time can make a comprehensive, accurate evaluation of gastric motility. At present, the contraction rate of gastric electrical slow wave can be described by surface electrode method to measure the electrogastrogram (Electrogastrogram, EGG) and other methods. But because of the low pass filter effect of body fat layer on the gastric electrical fast wave, gastric fast wave cannot be collected on the surface of body.

Gastric electromagnetic signal is a flow of electrons from the stomach cell membrane. Slow wave has low frequency, it is about $0.05 \mathrm{~Hz}$, fast wave has the higher frequency, it is about $1.3 \sim 2 \mathrm{~Hz}$ [2]. The attenuation is very small when gastric magnetic fast wave produced by gastric electrical fast wave pass the tissue, so this paper uses the giant magneto impedance
(Giant Magneto-Impedance effect, GMI) sensor array to obtain gastric magnetic signal, and then extract the key technology of gastric electrical fast information, this will play an important role in capturing the problem, which cannot detect the domain gastric fast wave signal from body surface, and it is a new methods to explore the function of stomach disease[3]. It is a very meaningful exploration.

\section{GASTRIC MAgNETiC Signal ACQUiSition AND PROCESSING SYSTEM BASED ON GMI}

There is DC and AC component of the output signal of the GMI sensor, the DC component has a great influence on the test results, it will cause very large baseline drift, so it must be treated. Eliminate DC link is added to the output end of the sensor. The next is the filtering and amplification link, the detection of weak signal must be filtered and amplified in the strong background noise. This is because the noise is far greater than the signal amplitude, the system added two stage low ratio amplify and filter after the sensor. First amplitude noise signal, then highly amplify the signal, this is because the noise is too strong, the amplifier useful signals at the same time, also led to the noise signal amplitude is too large, the amplifier will be in the saturated output working state, the useful signal would be weakened further, it also makes the subsequent filter to lose the due meaning. Gastric magnetic data signal acquisition system block diagram is shown in figure 1 .

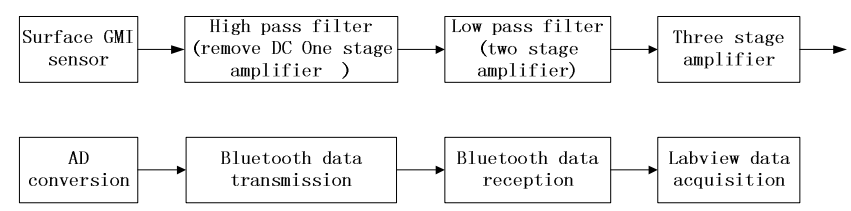

FIGURE I: DATA ACQUISITION AND PRE-TREATMENT OF GASTRIC MAGNETIC SIGNAL.

The output signals of amplifier is analog signals, need to A/D conversion, converted to analog signal and sent to the microcontroller and Bluetooth output. The signal from the Bluetooth, received by Bluetooth module PC machine, and transmits PC data processing module for processing, and display. The function gastric magnetic signal detection system GMI software platform to complete is: Based on Bluetooth data receiving, gastric magnetic signal de-noising and display of gastric magnetic signal. 
The sensor output signal needs filtering, this design adopt the band pass filter. Because the frequency of gastric electrical slow wave is $0.03 \mathrm{~Hz} \sim 0.05 \mathrm{~Hz}$, the frequency of gastric electrical fast wave is $1.3 \mathrm{~Hz} \sim 2 \mathrm{~Hz}$, considering the signal attenuation in the cut off frequency, the high pass cut-off frequency is set to $0.01 \mathrm{~Hz}$, and the low pass cut-off frequency is set to $2.5 \mathrm{~Hz}$. To eliminate the DC component, this design adopt high pass filter. High pass filter is the two rank SallenKey circuit structures. Low pass filter is to filter the signal output of the high pass filter to below $2.5 \mathrm{~Hz}$, filter using four order Butterworth filter, consists of two level Sallen-Key circuit.

The amplifier of gastric magnetic signal detection system based on GMI has the following characteristics: first, precision must be high. Gastric magnetic signal is very weak; any disturbance in the circuit will have a great effect on the signal acquisition. Second, small volume. Third, the large magnification. The signal between filter outputs, the useful signal must be $\mathrm{mV}$ level, so we need to enlarge big multiplier in order to ensure the work efficiency behind the signal acquisition system. Based on the above factors, the system uses AD620 as the amplifier.

The Bluetooth technology provides low cost and short distance wireless communication, Bluetooth can constitute the communication between PC and mobile devices, the gastric magnetic signal can be transferred from measuring and signal conditioning module close to PC machine, so as to realize the purpose of no cable connection. Enhance the system portability. The data acquisition system of the design is a Bluetooth wireless data transmission system based on MSP430AFE251 microcomputer. It has a 24 bit A/D conversion accuracy, meets the requirement of the system design, and the volume is smaller, has less pins, programming is very simple.

\section{Gastric Magnetic Signal Data AnAlysis Method}

Make the power spectrum analysis of the gastric magnetic signal, find out the interference of different frequency bands, and filter them. In this paper, the application of autoregressive spectral analysis results as shown in figure 2 :

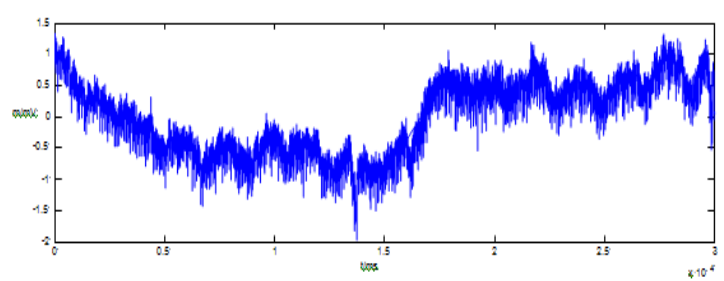

(a) The measured gastric magnetic wave

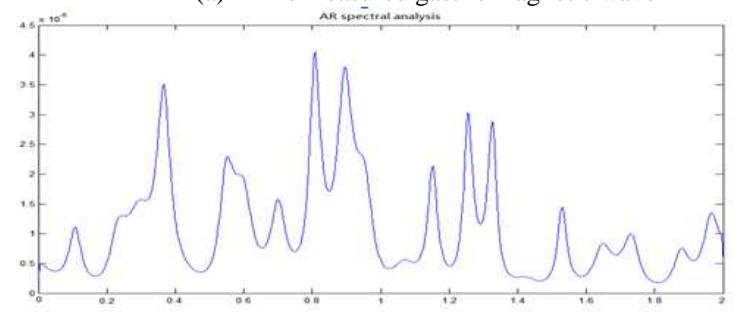

(b) AR spectral analysis

FIGURE II: MGG AND AR SPECTRUM ANALYSIS OF MGG.
As shown in Figure 2(a), it is the waveform for GMI in the coordinate $(2,3)$ point. It can be seen in the waveform, the signal of the sensor that go through the hardware filtering and amplification, the noise is also very big. Make AR spectrum analysis of it, which part of the $0 \sim 2 \mathrm{~Hz}$ as shown in Figure 2(b) shows the measured gastric magnetic signal autoregressive power spectrum analysis of the spectrum. It can be seen from the graph, the frequency has higher energy at $0.005 \mathrm{~Hz}, 0.05 \mathrm{~Hz}$, $0.4 \mathrm{~Hz}, 0.5 \mathrm{~Hz}, 0.8 \mathrm{~Hz}, 0.9 \mathrm{~Hz}, 1.15 \mathrm{~Hz}, 1.25 \mathrm{~Hz}, 1.3 \mathrm{~Hz}, 1.5 \mathrm{~Hz}$, and $1.95 \mathrm{~Hz}$ etc. Among them, $0.05 \mathrm{~Hz}$ is the gastric magnetic slow wave, $0.4 \mathrm{~Hz}$ is respiratory interference and motion interference with $0.005 \mathrm{~Hz}, 1.25 \mathrm{~Hz}$ is the heart of magnetic fluctuations, there are gastric magnetic fast signal between $1.3 \sim 2 \mathrm{~Hz}$, the wave in the other band may be outside disorderly magnetic interference. Visibly, gastric magnetic signal is very weak, be covered by a lot of noise. To remove these noises and get the accurate signal gastric magnetic signal, we need a suitable intelligent algorithm of weak signal extraction.

\section{Gastric Magnetic Signal De-Noising Algorithm BASED ON WAVELET ANALYSIS AND CHAOS THEORY}

This paper adopts wavelet threshold de-noising and chaos theory to remove the strong noise in gastric magnetic signal. Chaotic system is immune to the strong background noise, and wavelet theory has a powerful advantage in signal filtering and feature extraction. However, using single chaos system to denoise the signal will cause the measurement accuracy of misalignment, mainly because the output signal of the GMI sensor has many components, as the above noise perturbation mentioned, we know that signal phase trajectory is very rough, noise is stronger, the observed effect is worse. In order to solve this problem, this design combines the wavelet analysis and chaotic oscillator system, formed a joint detection system to identify the weak signal. Wavelet analysis can weaken the interference signal well, and highlight the characteristics of gastric magnetic signal; the accuracy of the detection of weak characteristic signal can be greatly improved. The specific process of signal de-noising is shown in figure 3 .

\begin{tabular}{|c|c|c|c|}
\hline $\begin{array}{c}\text { The measured } \\
\text { gastric magnetic } \\
\text { signal }\end{array}$ & $\begin{array}{c}\text { Wavelet } \\
\text { denoising }\end{array}$ & $\begin{array}{l}\text { Chaotic } \\
\text { system }\end{array}$ & $\begin{array}{c}\text { Output } \\
\text { Display }\end{array}$ \\
\hline
\end{tabular}

FIGURE III: THE FLOW CHART OF DE-NOISING AND DISPLAY PROGRAM OF MGG.

\section{A. De-Noising Methods of the Gastric Magnetic Signal Based on Wavelet Analysis}

Wavelet analysis is very powerful in filtering and feature extraction of signal. In the practical project, the signal content coming from GMI sensor is extremely rich, through the foregoing analysis of power spectrum, it can be seen, after the hardware filter and DC, many ingredients are still not filtered out, these components are close to gastric magnetic signal frequency, if these signals are into a chaotic system directly, it will have very adverse effects on test results. So this paper introduced the wavelet analysis, first filter the signal.

The wavelet transform of signal steps:

(1) Select wavelet scales a, compare the initial period of the gastric magnetic signal with the chose wavelet; 
(2) Apply CWT (a, b) to calculate the correlation degree of gastric magnetic signal and the selected wavelet, more similar the two are, the bigger CWT $(a, b)$ is. The results depend on the selected wavelet shape;

(3) Wavelet translation, mainly by changing the b value to realize, repeat 1,2 steps, until the completion of signal to complete a comparative analysis;

(4) Continue to stretch the wavelet, which increases the value of a, repeated $1 \sim 3$ step, analyze the next round of the signal;

(5) Repeat $1 \sim 4$ steps for all the scale factors, we can get large coefficient of the different scale a in the assessment of signal in different time intervals. These coefficients can reflect gastric magnetic analysis of projection signal on wavelet function, and we can use the grayscale represent it.

This paper chooses the threshold value filtering method. Threshold filtering method is mainly make wavelet decomposition of noise signal, classify wavelet coefficients of each layer coefficient of greater than and less than the one threshold, then use the wavelet coefficients after processing to reconstruct the signal, so as to achieve the purpose of filtering. This paper selected wavelet function for Haar function, decomposition of 3 layers. Measured gastric magnetic signals shown in Figure 2 (a) through wavelet de-noising experiment, obtained waveform is shown in figure 3 .

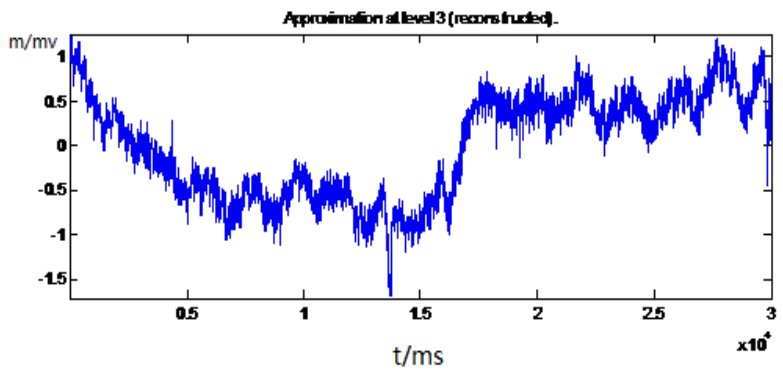

FIGURE IV: WAVEFORM OF GASTRIC MAGNETIC SIGNAL AFTER WAVELET DE-NOISING.

Only use soft threshold wavelet de-noising method is not very good to filter the interference signal, except the energy at $0.05 \mathrm{~Hz}$ has higher frequency, the other still has larger noise without filter. Slow wave of frequency has higher energy, this is not necessarily the gastric magnetic slow wave's energy, may be superimposed interference with other signals, use wavelet decomposition and reconstruction method singly, the extraction effect was not good. Therefore, we also need chaotic denoising.

\section{B. Gastric Magnetic Signal Detection Based on Chaos Theory}

Chaotic system is very sensitive to initial conditions, we can make gastric magnetic signal as the initial value, add to the chaos system, it will lead to changes in the nature of chaotic system, so it can extract gastric magnetic signal in strong noise background [4].

When driving signal and gastric magnetic signals are in the presence of frequency difference, the equations are as follows:

$$
\ddot{x}(t)+k \dot{x}(t)-x^{3}(t)+x^{5}(t)=f \cdot \cos \omega t+A \cos ((1+\Delta \omega) t+\varphi)
$$

In the formula, $\Delta \omega_{\text {is }}$ the angular frequency difference of the driving signal and the stomach in the magnetic signal, $\mathrm{A}$ is gastric magnetic signal amplitude, $\varphi_{\text {is the phase of gastric }}$ magnetic signal. Type (1) in the right end can transform:

$$
f \cos (t)+A \cos ((1+\Delta \omega) t+\varphi)=F(t) \cdot \cos (t+\theta(t))
$$

Among them,

$$
\begin{gathered}
F(t)=\sqrt{f^{2}+2 f \cdot A \cos (\Delta \omega t+\varphi)+A^{2}} \\
\theta(t)=\arctan \left(\frac{A \sin (\Delta \omega t+\varphi)}{f+A \cos (\Delta \omega t+\varphi)}\right)
\end{gathered}
$$

When $\Delta \omega$ is zero, the amplitude of gastric magnetic signal is generally less than $\mathrm{f}$, it shows that in (4), the value of $\theta(t)$ is very small. Experiments show that, the phase in $(-\pi / 2, \pi / 2)$ range, the detection of weak signals basically can be observed through the changes of phase plane to achieve.

When $\Delta \omega$ is not zero, known by type (3), $\mathrm{F}(\mathrm{t})$ will alternate in the interval $(f-A, f+A)$, in the intermittent chaos. When $\Delta \omega$ is small, the change process of $\mathrm{F}(\mathrm{t})$ will slow to phase change, the system will respond very well to the driving force of the slow change. This leads to the periodic and chaotic phenomena has obvious watershed, when the system has small frequency difference, $\mathrm{F}(\mathrm{t})$ will change small near the $\mathrm{f}$ value, the system will be doing intermittent chaotic motion in period of $\Delta T=2 \pi / \Delta \omega$. Thus, we can obtain gastric magnetic signal frequency through the detection of $\Delta T$. However, the need to pay attention to is $\Delta \omega$ and the frequency of the system drive ratio cannot exceed 0.04 , otherwise intermittent chaotic phenomenon will not be recognized, because the $F(t)$ changes too fast. Because the amplitude of gastric magnetic signal is very small, oscillator model can therefore be used to finish the measurement. When frequency difference is less than or equal to 0.02 , oscillator model for detection of arbitrary frequency is:

$$
\begin{aligned}
& \ddot{x}(t)+\omega \cdot k \cdot \dot{x}(t)-\omega^{2} \cdot x^{3}(t)+\omega^{2} \cdot x^{5}(t)=\omega^{2} \cdot f \cos (t) \\
& +\omega^{2} \cdot\left\{A \cos \left(\omega^{*} \cdot t\right)+\sigma \cdot \operatorname{randn}(t)\right\}
\end{aligned}
$$

In the formula, $\left\{A \cos \left(\omega^{*} \cdot t\right)+\sigma \cdot \operatorname{randn}(t)\right\}$ is the detection of weak signal and noise signal superposition. Applied in this design, it can be changed into:

$$
\ddot{x}(t)+\omega \cdot k \cdot \dot{x}(t)-\omega^{2} \cdot x^{3}(t)+\omega^{2} \cdot x^{5}(t)=\omega^{2} \cdot f \cos (t)+\omega^{2} \cdot M G G(6)
$$

Where MGG represents the measured data of gastric magnetic signal.

Therefore the steps of using chaos theory for treatment of gastric magnetic signal are:

(1) Confirm the chaotic oscillator detection system to be intermittent chaotic system for Duffing model. The specific form of chaotic oscillator equation is: $\ddot{x}(t)+\omega \cdot k \cdot \dot{x}(t)-\omega^{2} \cdot x^{3}(t)+\omega^{2} \cdot x^{5}(t)=\omega^{2} \cdot f \cos (t)+\omega^{2} \cdot M G G$ 
determine the most favourable parameters to gastric magnetic signal detection.

(2) Establish a one-dimensional array, contains all the frequency data to be detected, $\Omega=\left\{\omega_{1}, \omega_{2}, \cdots, \omega_{\mathrm{n}}\right\}$, these values are determined using arithmetic or geometric form. Attention is required to ensure that between adjacent frequency point frequency difference is always less than or equal to 0.02 , so as to ensure the appearance of the intermittent chaos, or gastric magnetic signal cannot be measured.

(3) Input the gastric magnetic signals and $\Omega$ to the chaotic system, if the gastric magnetic signals frequency is equal to $\omega^{*}=\omega$.

, it can detect gastric magnetic signal frequency through the phase change trajectories of chaos oscillator system from chaotic state to the large-scale periodic state plane; if $\omega_{i}<\omega^{*}<\omega_{i}+1,\left(\omega_{i}, \omega_{i+1} \in \Omega\right)$

chaos intermittency of chaos oscillator system to determine the existence of weak signal, the frequency difference $\Delta \omega$ is determined by $\Delta T=2 \pi / \Delta \omega$, use the frequency difference to calculate the gastric magnetic signal frequency value.

(4) When not observed the chaotic intermittency of chaos oscillator, or no periodic motion occurs, we need to change the value of $\Omega$ appropriately for next detection.

(5) When detected the frequency of gastric magnetic signal, we can estimate the amplitude. The specific method is: change the chaotic oscillator system, by reducing the threshold $f$, the value reduced is equal to the amplitude of gastric magnetic signal. Because the amplitude of gastric magnetic signal is very small, it needs to be multiplied by a fixed proportion coefficient before inputting to chaotic system. Select $\mathrm{f}=1.973, \mathrm{k}=0.5$, set $\omega=2.02$, put the data of wavelet de-noising into MGG module, we can get gastric magnetic wave. Phase contrast of chaotic system before joining MGG (a) and MGG (b) is shown in the figure, figure 5 is the gastric magnetic signal fragment after chaotic system de-noising. Due to the measurement time is short, the gastric magnetic signal does not reflect the slow wave cycle $0.05 \mathrm{~Hz}$. But you can also see a clear peak wave superpose on gastric magnetic slow wave, namely gastric magnetic fast waveform, visible system can get stomach fast wave and gastric slow wave at the same time. Extend the measurement time to $20 \mathrm{~min}$, the de-noising wave is shown in Figure 6, accord with the characteristics of gastric slow and fast wave.

Analyzing the power spectrum of the gastric magnetic signal, as shown in Figure 7, visible after the filter of the chaos, the power of two frequency component gastric slow wave and fast wave in magnetic is large, the signal with high SNR, has good effect of de-noising. Combining chaos theory and wavelet theory to de-noise gastric magnetic signal is better, in the signal we obtained, the gastric magnetic fast and slow wave have high content of possession, it illustrated that we can get gastric magnetic signal with high SNR by this algorithm.

Through the comparison of the effect of wavelet de-noising and wavelet chaotic combined reduction, it can be seen, singly add wavelet analysis in the gastric magnetic signal detection has poor effect, gastric electrical slow wave and gastric electrical fast wave cannot be clearly demonstrated, while introducing chaos system is very effective to improve SNR of gastric magnetic signal.

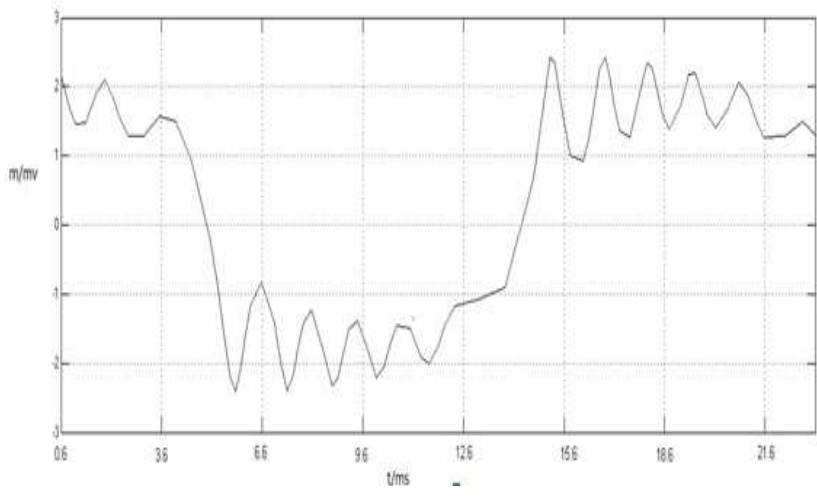

FIGURE V: FRAGMENT OF MGG AFTER DE-NOISING.

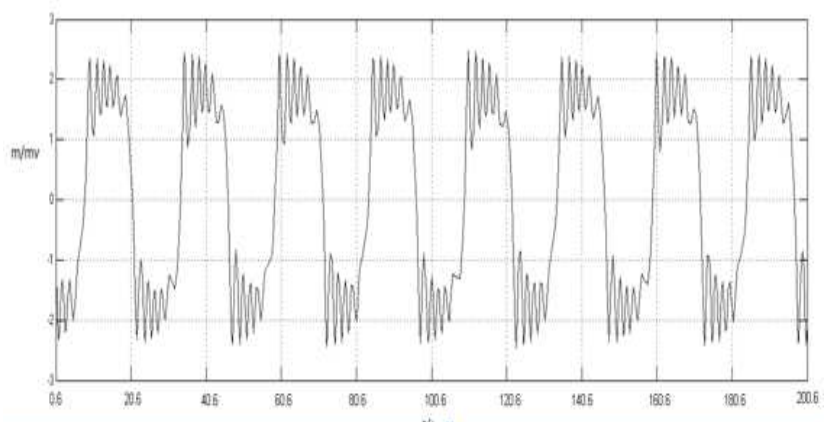

FIGURE VI: MGG AFTER DE-NOISING.

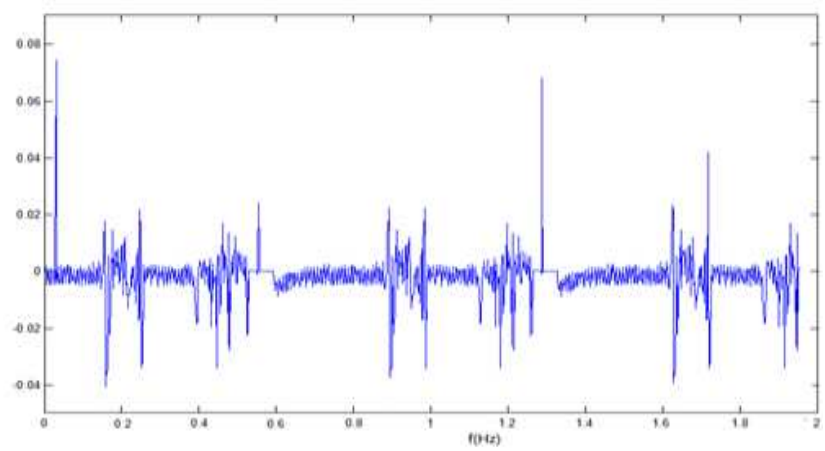

FIGURE VII: THE AR POWER SPECTRUM OF MGG AFTER DENOISING.

\section{SUMMARY AND OUTLOOK}

This paper designed the gastric magnetic device detection system based on GMI sensor, the creative use of small first and large second amplification mode, guarantee the amplifier is not in saturated state, and eliminated the DC component in the process, improved the precision of the measuring system. Use the chaotic theory for the detection of gastric electromagnetic signals firstly, put forward the algorithm combined wavelet analysis and chaos theory, improved the de-noising effect of gastric magnetic signal. 


\section{ACKNOWLEDGMENTS}

This research is supported by Shenyang city science and technology project of China-Research on detection and recognition of gastric magnetic signal and its application in the diagnosis of gastrointestinal functional diseases (Grant No. F13-053-2-00).

\section{REFERENCES}

[1] S Somarajan, ND Muszynski, C Obioha, WO Richards, and LA Bradshaw. Biomagnetic and bioelectric detection of gastric slow wave activity in normal human subjects-a correlation study.PhysiolMeas, 33(7), pp. 1171-1179, 2012.

[2] L. Alan Bradshaw, Leo K. Cheng, William O. Richards, and Andrew J. Pullan. Surface Current Density Mapping for Identification of Gastric Slow Wave Propagation.IEEE Transactions on Biomedical Engineering, 56(8), pp. 31-39, 2009.

[3] C.Tannous, J. Gieraltowski. Giant magneto-impedance and its applications.Journal of materials science: Materials in electronics, 15(4), pp. 25-33, 2004.

[4] Zhao Zhan, Li Yaoming, Cheng Jin, Xu Yunfeng. Current sensor utilizing giant magneto-impedance effect in amorphousribbon toroidal core and CMOS inverter multivibrator.Sensors and Actuators, 137 (7), pp. 64-67, 2007. 